Document downloaded from:

http://hdl.handle.net/10251/50308

This paper must be cited as:

Calderón García, PA.; Glisic, B. (2012). Influence of mechanical and geometrical properties of embedded long-gauge strain sensors on the accuracy of strain measurement. Measurement Science and Technology. (23):1-15. doi:10.1088/0957-0233/23/6/065604.

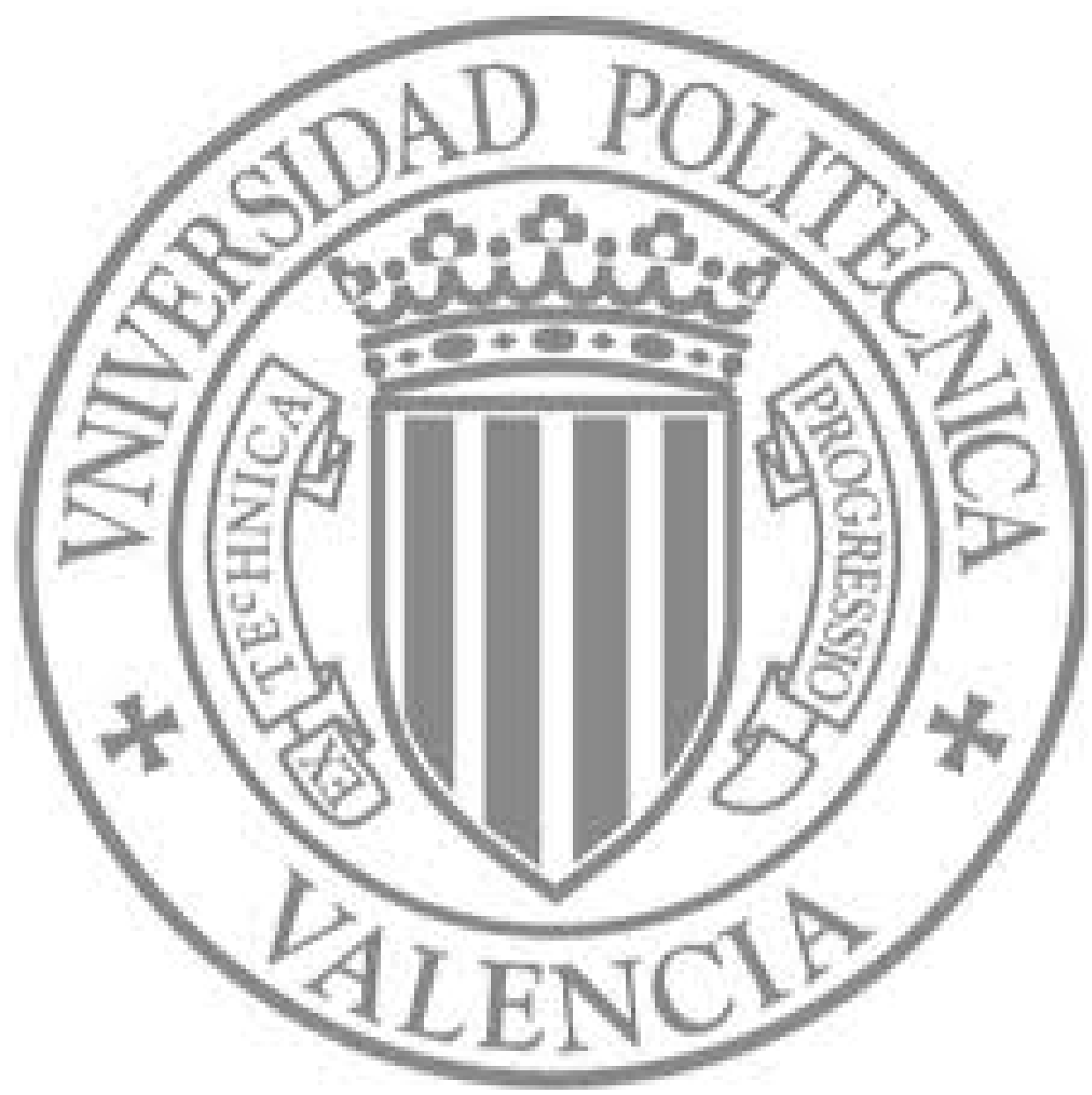

The final publication is available at

http://dx.doi.org/10.1088/0957-0233/23/6/065604

Copyright

IOP Publishing: Hybrid Open Access 
Influence of mechanical and geometrical properties of embedded long-gauge strain sensors to the accuracy of the strain measurement

\section{Influence of mechanical and geometrical properties of embedded long-gauge strain sensors to the accuracy of the strain measurement}

3

Pedro A. Calderón

Universitat Politècnica de Valencia, Camino de vera s/n, 46022 Valencia, Spain

e-mail: pcaldero@upv.es, phone: + 34963877562 (ext.:75620).

\section{Branko Glisic (CORRESPONDING AUTHOR)}

Princeton University, E330 EQuad, Princeton, NJ 08540, USA

e-mail: bglisic@ princeton.edu, phone: +16092588278.

E-mail: bglisic@princeton.edu

Abstract: In many civil and geotechnical applications it is of interest to monitor the strain deep inside the structure, consequently it is necessary to embed the sensors into the structure's material. Construction and geotechnical materials, such as concrete and soil, can be affected by local defects, e.g. cracks, air pockets, and inclusions. To monitor these materials at a structural level it is necessary to use long-gauge sensors. As the sensor has to be embedded in the host material, its presence causes perturbation of the strain field and influence the accuracy of the strain measurement. The aim of this research was to identify the critical parameters that influence the accuracy of the strain measurement, to study how these parameters affect the accuracy, and to give the recommendations for the sensor users. The study was based on finite element analysis and all involved materials were assumed to have the Möhr-Coulomb elastic-perfectly plastic behavior. A suitability of the numerical model for the analysis was verified using the experimental results of two cases reported in the literature and one on-site application. The study revealed that the most important parameters that influence the accuracy of the strain measurement are the goodness of interaction (strain transfer) between the host material and the anchor pieces of the sensor, the ratio between equivalent Young modulus of the sensor and the Young modulus of the host material, the radius of the anchor piece, and the gauge length. The numerical model and parametric study are presented in details along with practical recommendations.

Keywords: long-gauge sensors, accuracy of strain measurement, embedded fiber optic sensors, geotechnical applications, concrete structures 
Influence of mechanical and geometrical properties of embedded long-gauge strain sensors to the accuracy of the strain measurement

\section{Introduction}

2 With reference to their spatial disposition, sensors are classified as discrete or point sensors, or as continuous or distributed sensors. Discrete sensors measure relative displacement or average strain between two predefined points. The distance between these two points is called the gauge length of the sensor. With respect to the gauge length, the sensors are conventionally classified in two groups: shortgauge and long-gauge sensors. Traditional sensors, such as strain gauges [1], belong to the group of shortgauge sensors. Depending on their type, packaging, and construction material of monitored structure, strain sensors based on vibrating wires or optical fibers can function as short-gauge or as long-gauge sensors $[1,2]$. The availability of long-gauge sensors $[2,3,4]$ has brought new possibilities for structural health monitoring and they were proven to be applicable for monitoring at a global structural level [5]. In many civil and geotechnical applications it is of interest to monitor strains deep inside the structure, consequently it is necessary to embed the sensors into the structure's material. In these cases the packaging of the sensor must provide protection for the sensing element. This condition implies the use of mechanically robust materials for the sensor packaging, such as stainless steel, reinforced or nonreinforced polymers, etc. On the other hand, in order to provide with an accurate measurement, (1) a good interaction between the sensor and the structure must be guaranteed, i.e., the strain must be transferred from the structure to the sensor within acceptable error limits (ideally, strain should be fully transferred from the host structure to the sensor) and (2) the sensor presence must not perturb the strain field of the monitored structure, i.e. the packaging of the sensor must be non-intrusive. Hence, the packaging should be robust enough to provide for a safe embedding of the sensor, but in the same time it should be soft enough in order not to perturb strain field in the host structure and to guarantee good strain transfer from the structure to the sensor. As mechanically robust materials are usually stiff, the packaging can significantly contribute to overall stiffness of the sensor. Consequently, the applicability of the sensor for embedding in construction materials depends on mechanical and geometrical properties that have to be compatible with the material of the monitored structure.

The accuracy of measurement depends also on the strain transfer from the packaging to the sensing element. However, the imperfection of the strain transfer from the packaging to the sensing element is well understood and presented in literature (e.g. [6],[7], etc.), and the resulting errors are, in practice, usually minimized by adjusting the gauge factor of the sensor during the manufacturing (i.e., calibration of the sensor). That is the reason why this type the error is not studied in this research, and the focus is made to strain transfer from the host material to the packaging.

In spite of its importance, not many published studies have been carried out to examine in details the influence of mechanical and geometrical properties of the sensor to the accuracy of the strain measurement. Torres et al. [8] have studied it for surface short gauge sensors, determining how even 
Influence of mechanical and geometrical properties of embedded long-gauge strain sensors to the accuracy of the strain measurement

1 small differences in mechanical or geometrical properties of its components may induce important errors

2 in the readings of a fiber optic sensor. Kesavan et al. [9] studied behaviors of two different fiber optic sensors embedded in hardened concrete. The first sensor had stainless steel (stiffer) packaging, while the second had composite (softer) packaging. Both were compared with surface mounted strain gauges, and in the case of stainless steel packaged sensor a bigger discrepancy was measured. Glisic [10] compared a "stiff" and a "soft" long-gauge fiber optic sensors embedded in fresh concrete, and noticed important differences before the hardening of the concrete, but after the hardening both sensors measured the same deformation. Azenha et al. [11] obtained similar results with embedded vibrating wire sensors as well. All the above presented researches provide with important contribution in the area. However, they did not study the influence of the mechanical and geometrical properties to the accuracy of long-gauge sensors in a systematic manner, but rather on a case-by-case basis. In this paper the influence of mechanical and geometrical properties to the accuracy of long-gauge sensors is examined systematically taking into account mechanical properties of the host material, mechanical properties of the sensor, geometrical properties of the sensors namely the gauge length and the size of anchor piece, and mechanical properties of the interface between the anchor piece and the host material. First the finite element (FE) model was built, then the model was validated using experimental data, and finally, a parametric study was performed, influence of mechanical and geometrical properties to the accuracy of long-gauge sensors is assessed and practical guidelines for real applications are derived.

\section{Embedded Long-gauge Sensors}

Measurements obtained with short-gauge sensors are in general different from measurements obtained with long-gauge sensors, and the main difference is in the information contained in the measurement. A short-gauge sensor provides with information on strain at a point and allows understanding of structure's behavior at local material level. A long-gauge sensor provides with an average strain value over a range of points, which is information useful to understand global, structural behavior [5].

Construction and geotechnical materials, such as concrete and soil, can be affected by local defects, such as cracks, air pockets, and inclusions. All these defects introduce discontinuities in the mechanical material properties at a micro and meso-level. More indicative for structural behavior, however, are material properties at the macro-level. For example, although reinforced concrete consists of hardened cement paste, matrix filled with aggregates of different sizes, and steel reinforcing bars, reinforced concrete structures are mainly analyzed at the macro level as built of a virtually homogenous material cracked reinforced concrete [12]. Therefore, for structural monitoring purposes it is necessary to use sensors that are insensitive to material discontinuities observed at the micro- and meso-levels while still providing reliable measurements at the macro-level. In other words, it is not of interest to know the exact 
Influence of mechanical and geometrical properties of embedded long-gauge strain sensors to the accuracy of the strain measurement

1 strain in each component of the material - hardened cement paste, aggregate, and steel - but to evaluate the behavior of the resulting material as a whole. The value of a measurement performed using longgauge sensor represents an average strain value along the sensor's gauge length which is commonly attributed to the midpoint of the sensor. In an inhomogeneous material, the gauge length of a deformation sensor can cross several discontinuities that influence the measurement and its interpretation. This is illustrated in Figure 1 and described in Equation (1) [5], which shows that for sensor whose gauge length is delimited by anchor pieces $A$ and $B$, the measured strain $\varepsilon_{C, s}$ depends on the strain distribution $\varepsilon_{x, s}(x)$ between anchor pieces $A$ and $B$, the number and the magnitude of dimensional changes of discontinuities $\Delta w_{d, i}$ (e.g. crack openings, inclusions, etc.) between $A$ and $B$, and the gauge length $l_{s}$.

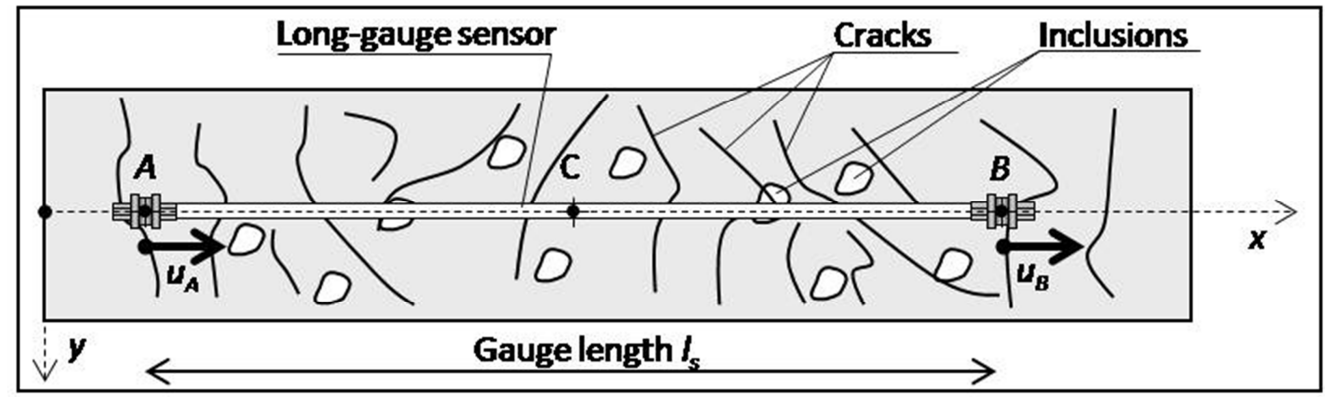

Figure 1. Long-gauge sensor in an inhomogeneous material (courtesy of SMARTEC SA).

$$
\varepsilon_{C, s}=\frac{\Delta l_{s}}{l_{s}}=\frac{1}{l_{s}} \int_{x_{A}}^{x_{B}} \varepsilon_{x, s}(x) \mathrm{d} x+\frac{1}{l_{s}} \sum_{i} \Delta w_{d, i}
$$

A short-gauge deformation sensor has a gauge length shorter than the distance between two discontinuities or comparable to the dimensions of the inclusions in the material monitored. Therefore, the measurement performed with short-gauge sensors is strongly influenced by local defects; it provides information related to local material properties and is not suitable for global structural monitoring. A long-gauge deformation sensor is by definition a sensor with a gauge-length several times longer than the maximal distance between discontinuities or the maximal diameter of inclusions in a monitored material. For example, in the case of cracked reinforced concrete, the gauge length of a long-gauge sensor is to be several times longer than both the maximum distance between cracks and the diameter of inclusions. The main advantage of this measurement is in its nature: since it is obtained by averaging the strain over long measurement basis, it is not influenced by local material discontinuities and inclusions, and the measurement contains information related to global structural behavior.

Based on the discussion presented in this section, division between short and long-gauge sensor depends on the properties of the material of the monitored structure. Measures [2] proposes the length of $50 \mathrm{~mm}$ as the limit between short and long gauge lengths. This division can be acceptable for homogeneous materials, such as steel, or for some inhomogeneous material with very small distances between discontinuities and very small inclusions, such as mortar. However, the proposed division cannot be 
Influence of mechanical and geometrical properties of embedded long-gauge strain sensors to the accuracy of the strain measurement

1 applicable for other inhomogeneous materials, such as concrete, where the aggregate size can be as big as $232 \mathrm{~mm}$, and the distance between the structural cracks 100 to $200 \mathrm{~mm}$. Thus a simple division between short- and long-gauge sensors cannot be established, and it depends on the host material properties (e.g. $50 \mathrm{~mm}$-long sensor is "long-gauge" if applied to steel, but it is "short-gauge" if applied to concrete).

Several types of long-gauge sensors developed during the last decade and a half $[2,3,4]$ were designed for embedding in concrete and soils. Thus, they can provide with direct measurements of the strain in the interior of structure or soil. For structures, the measurements performed with embedded sensors are free of errors that may be induced in the case the sensors are installed on the surface of the structure (e.g. due to thermal influence to sensor anchoring points, differences in strain at surface and in interior of the structure, non-linear distribution of strain in structure's interior, etc.). For soils, the surface installed sensors cannot provide with detailed information regarding interior, and the embedded sensors provide with unique solutions to assess the internal strain in soils. An example of long-gauge sensors during the embedding in the concrete is shown in Figure 2.

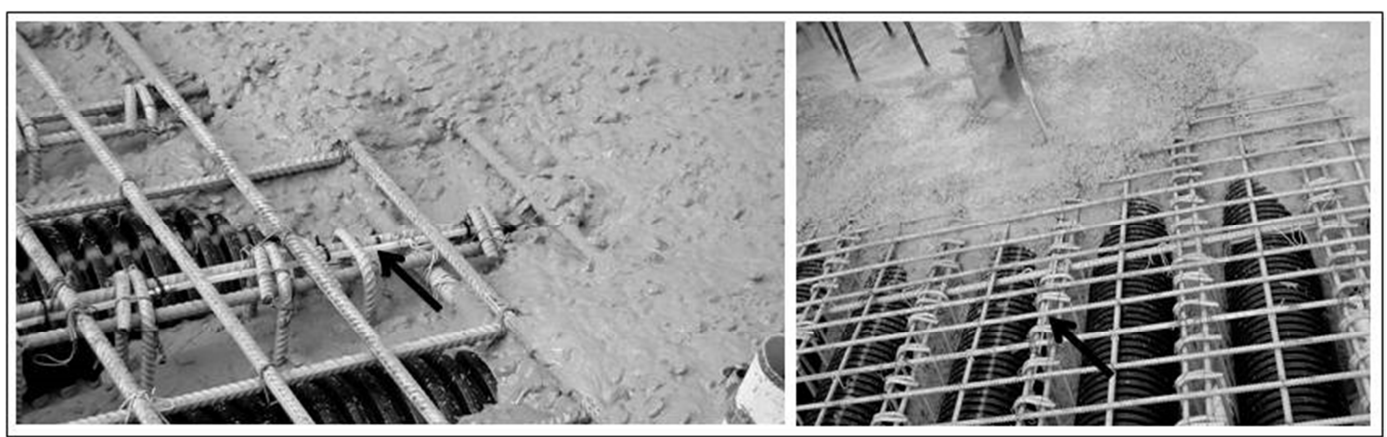

Figure 2. Embedding in concrete of long-gauge sensors (sensor indicated with arrow).

The long-gauge sensors have, in general, two types of errors that are inherent to their geometrical and mechanical properties. The first is inherent to sensors gauge length and variability of the strain field between the sensors anchoring points. The analysis of this error exceeds the contents of this paper and can be found in literature [5,13]. The second type of error is related to the level of the strain transfer from the host material to the sensor, which mainly depends on the size of anchoring pieces of the sensor, the gauge length, and the mechanical properties of both the sensor and the host material. This second type of error is the topic of this paper and is in detail analyzed and presented in the next sections.

\section{Numerical Model}

In order to analyze how the geometrical and mechanical properties of both the packaging and the host material affect the strain measurements, a study based on validated FE modeling was performed. The 
Influence of mechanical and geometrical properties of embedded long-gauge strain sensors to the accuracy of the strain measurement

software used for this study was PLAXIS 2D-Ver. 8.2 [14]. In order to simulate various possible scenarios of the behavior of the sensors embedded in host material, the following assumptions were made:

a) Most of long-gauge sensors available on the market (e.g. see Figure 3):

- are composed of cylindrical bodies and they are axially symmetric with respect to their center-line;

- are additionally symmetric with respect to the plane normal to center-line at the middle of the sensor;

- have gauge length several times larger than the cross-sectional dimensions;

The above three features of the long-gauge sensors allow to reduce the analysis from complex threedimensional space, to simpler, two-dimensional half-space as shown in Figure 3 and Section 3.1.

b) The host material can be modeled using Möhr-Coulomb constitutive equations for elastic, perfectly plastic material [15].

c) Mechanical interaction at the interface between the sensor and the host material can be modeled using so-called "interface" elements and Möhr-Coulomb constitutive equations.

\subsection{Description of the Finite Elements, Boundary Conditions, and Loads}

To analyze the behavior of embedded sensor, it is assumed that the host material is subjected to uniform uni-directional load. In the general case, the presence of the sensor perturbs the strain field, but for points "far enough" from the sensor, in both vertical and horizontal directions, the perturbation can be neglected [16]. Thus, the volume of the host material under analysis can be reduced to include the part which is perturbed and a small neighboring zone of non-perturbed material in all directions. The dimensions of the specimen were determined based on a sensitivity study and a radius of $1 \mathrm{~m}$ and height of $4 \mathrm{~m}$ (half-height of $2 \mathrm{~m}$ ) were chosen. As the sensor is axially symmetric, the analyzed volume is also axially symmetric and has a shape of cylinder. The external boundary of the cylinder is in non-perturbed zone which is only subjected to vertical unidirectional strain (no displacements in horizontal plane) and, therefore, it can be considered as laterally confined. Due to symmetry of the applied load and the symmetry of the sensor with respect to plane normal to the axis of the sensor, only one half of the cylinder was analyzed. General geometrical properties of the sensor and host material used in this study are shown in Figure 3.

Due to axial symmetries, the host material and the sensor are analyzed in two-dimensional confined space, as shown in Figure 3. They are modeled using 15-node triangular elements. The element provides a fourth order interpolation for displacements and the numerical integration involves twelve Gauss points (stress points). Each node has two translational degrees of freedom per node ( $x$ and $y$ direction). The finite element mesh used in the parametric study has 1010 elements, 8327 nodes and 12120 stress points. The mesh has been thoroughly refined at the sensor and its vicinity. The mesh is shown in Figure 3. 
Influence of mechanical and geometrical properties of embedded long-gauge strain sensors to the accuracy of the strain measurement

1 Uniform vertical loads are applied at the top boundary. In the parametric study presented later in the

2 paper, the applied loads range from zero to the unconfined compressive strength of the material. Since the

3 points on the vertical boundary are in non-perturbed zone, they can experience only vertical

4 displacements and thus have horizontal constraints only. Bottom boundary is the plane of symmetry of

5 the sensor and consequently, it has vertical constraints only.

6 The interaction between the sensor and the host material is modeled by "interface" elements. For a 15-

7 node triangular element the corresponding "interface" elements is defined by five pairs of nodes. In the

8 FE formulation the coordinates of each node pair are identical, although the interface has an assigned

9

10

11

12

13

14

15

16

17

18

19

20 "virtual thickness", which is necessary to define the material properties of the interface.

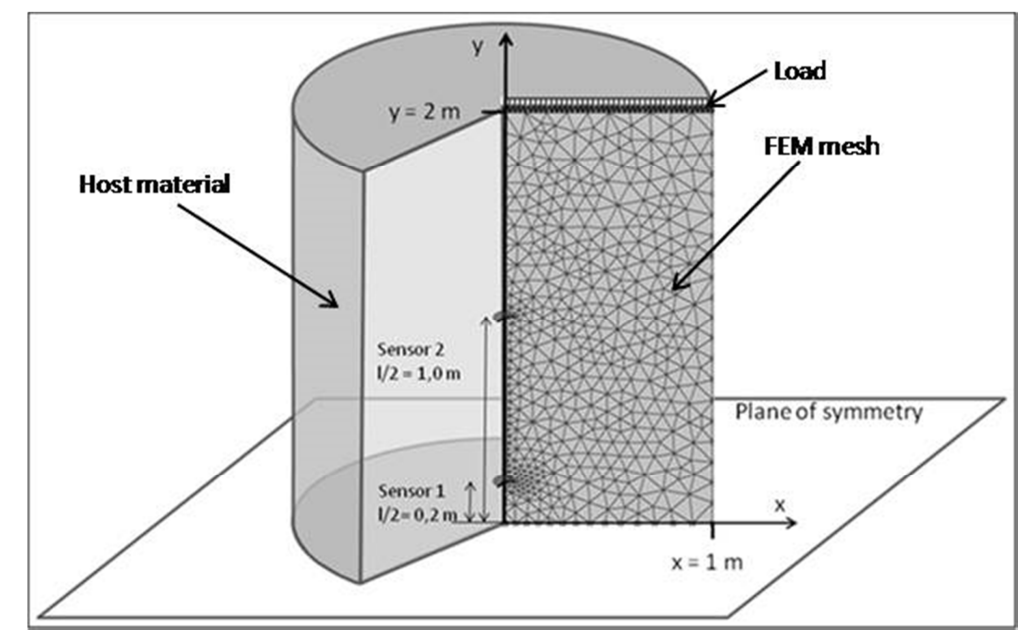

Figure 3. Geometrical properties and mesh of general FE model used in the parametric study.

The geometrical properties presented in the next sub-sections are used in the parametric study presented in Section 4. For validation of the general FE model presented in Appendix A, the dimensions, boundary conditions, and loads were adjusted to real values and conditions, as found in the tests.

\subsection{Modeling the Host Material and the Sensors}

The model of each material was created depending on the real material that the model intends to represent. Five different materials were considered for host material in the parametric study, ranging from a stiff soil to a hardened concrete. The plastic behavior of the materials was characterized using MöhrCoulomb constitutive equations for elastic, perfectly plastic material. The model involves five parameters, namely Young modulus $E$, Poisson's ratio $v$, the cohesion $c$, the friction angle $\varphi$, and the dilatancy angle $\psi$. Dilatancy angle was considered equal to zero, which is typical value for the materials under 
Influence of mechanical and geometrical properties of embedded long-gauge strain sensors to the accuracy of the strain measurement

1 consideration. According to the Möhr-Coulomb's criterion, the unconfined compressive strength of the material, $f^{\prime}{ }_{c 0}$, can be derived from the values of the cohesion and the friction angle, see Equation (2):

$$
f_{c 0}^{\prime}=\frac{2 \cdot c \cdot \cos \varphi}{1-\sin \varphi}
$$

The Young modulus was related to the unconfined compressive strength of the material. Equation (3) [17] was used in those instances where the material is considered to represent a "concrete":

$$
E_{c i}=E_{c 0} \cdot\left(f_{c} / f_{c m 0}\right)^{1 / 3}
$$

where $E_{c i}$ is the elastic modulus, $f_{c}$ is the compressive strength, and $f_{c m o}, E_{c 0}$ have values as defined in CEB-FIB Model Code 90 [17]: $f_{c m 0}=10 \mathrm{MPa} ; E_{c 0}=21.5 \mathrm{GPa}$.

Equation (4) was used for materials considered as "soils":

$$
E_{c i}=k \cdot f^{\prime}{ }_{c 0}
$$

where $k=250$ is typical value for low to medium plasticity cohesive soils with medium overconsolidation ratio, and the relationship between $f^{\prime}{ }_{c 0}$ and $E_{c i}$ can be found e.g. in [18].

The mechanical parameters used to model the host materials are shown in Table 1 for both the verification of the general FE model given in Appendix A, and the parametric study presented in Section 4.

Table 1. Mechanical parameters of the host materials used in the FE models.

\begin{tabular}{|l|l|l|l|l|l|l|}
\hline Id. & Host material & $\mathbf{c}[\mathbf{M P a}]$ & $\varphi\left[{ }^{\circ}\right]$ & $\mathbf{f}_{\mathrm{c} 0}[\mathrm{MPa}]$ & $\mathbf{E}_{\mathrm{ci}}[\mathbf{G P a}]$ & $\mathbf{v}$ \\
\hline
\end{tabular}

Used to verify the FE model (Appendix A)

\begin{tabular}{|c|l|c|c|c|c|c|}
\hline $\mathbf{1}$ & Kesavan et al. [9] & 24.90 & 30 & 86.26 & 44.1 & 0.20 \\
\hline $\mathbf{2}$ & Leng et al. [19] & 9.30 & 30 & 32.22 & 31.8 & 1.20 \\
\hline 3-a & Streicker bridge fresh concrete, lower limit & 0.05 & 25 & 0.14 & 0.025 & 0.30 \\
\hline 3-b & Streicker bridge fresh concrete, upper limit & 0.03 & 25 & 0.10 & 0.036 & 0.30 \\
\hline
\end{tabular}

Used in the parametric study (Section 4)

\begin{tabular}{|c|l|c|c|c|c|c|}
\hline a & Stiff soil (e.g. well compacted low plasticity clay) & 0.20 & 30 & 0.69 & 0.2 & 0.25 \\
\hline b & Hard soil / Soft rock (e.g. weak mudstone) & 1.50 & 30 & 5.20 & 1.3 & 0.22 \\
\hline c & Concrete in initial phase of hardening & 0.20 & 30 & 0.52 & 8.0 & 0.20 \\
\hline d & Low strength concrete & 3.20 & 30 & 11.02 & 22.2 & 0.20 \\
\hline e & Concrete & 5.90 & 30 & 20.44 & 27.3 & 0.20 \\
\hline
\end{tabular}

The sensors are assumed to have two qualitatively different components: anchor pieces at its ends and a straight bar, which packages the sensor. It is estimated that the sensor measures the average strain between the two opposite edges of the bar. The anchor pieces are assumed to be made of steel, while different materials were chosen for the bar. All sensor components were assumed to have a linear elastic 
Influence of mechanical and geometrical properties of embedded long-gauge strain sensors to the accuracy of the strain measurement

1 behavior. The geometrical and the mechanical properties of sensors used for the verification of the FE model in Appendix A were those of the real sensors used in the tests. However, for parametric study presented in the next section, an equivalent sensor is used with the assumed geometrical properties as follows:

- Anchor piece has a shape of disc with thickness of $0.01 \mathrm{~m}$, which ensured that the anchor piece is rigid relative to the host material; radii of 0.02 and $0.05 \mathrm{~m}$ were studied.

- Bar has a shape of cylinder with diameter of $8 \mathrm{~mm}$; to simplify the modeling and the analysis, the diameter of the bar was constant in the study; two different gauge lengths were studied, $0.4 \mathrm{~m}$ and 2.0 $\mathrm{m}$ (in the FE model half-lengths of $0.2 \mathrm{~m}$ and $1.0 \mathrm{~m}$ respectively were represented due to symmetry). In reality there may be a large variety of possible geometrical properties of the sensor, and analyzing all of them would be time consuming and inefficient. In addition it would be difficult and impractical to propose general guidelines applicable to a specific real sensor. That is why the equivalent sensor was introduced and analyzed in parametric study. Algorithm is developed on how to convert any real sensor into equivalent sensor (see further text and Expression 5). Hence, the analysis of equivalent sensor makes the research results universally applicable to any specific real sensor.

Four different types of sensor were considered in the parametric study. For all types of sensors the anchor pieces were assumed to be made of steel with Young modulus of $200 \mathrm{GPa}$, while the Young modulus of the bar was varied. Three FE models represent the real sensors as used in validation tests. These sensors feature a wide range of mechanical properties as presented in Table 2. An imaginary sensor with intermediate bar stiffness was added in order to fill the gap in the stiffness range, see Table 2.

Table 2. Description of the real sensors considered in the models.

\begin{tabular}{|c|l|c|c|c|c|c|c|c|}
\hline \multicolumn{1}{|c|}{ Description } & Materials & $\begin{array}{c}\mathbf{E}_{\mathbf{s}} \\
{[\mathbf{G P a}]}\end{array}$ & $\begin{array}{c}\mathbf{O D} \\
{[\mathbf{m m}]}\end{array}$ & $\begin{array}{c}\mathbf{I D} \\
{[\mathbf{m m}]}\end{array}$ & $\begin{array}{c}\mathbf{A}_{\mathbf{s}} \\
{\left[\mathbf{m m}^{2}\right]}\end{array}$ & $\begin{array}{c}\mathbf{A}_{\mathbf{o}} \\
{\left[\mathbf{m m}^{2}\right]}\end{array}$ & $\begin{array}{c}\mathbf{E}_{\mathbf{s}} \mathbf{A}_{\mathbf{s}} \\
{[\mathbf{k N}]}\end{array}$ \\
\hline \multirow{2}{*}{1} & $\begin{array}{l}\text { (Gl) - SMARTEC sensor (Glisic } \\
[10])\end{array}$ & Polyethylene & 1 & 8 & 6 & 22.0 & 50.3 & $\mathbf{2 2 . 0}$ \\
\hline 2 & (In) - Intermediate sensor* & Brass & 103 & 8 & 7.6 & 4.9 & 50.3 & $\mathbf{5 0 4 . 8}$ \\
\hline 3 & (Le) - Sensor by Leng et al. [19] & Steel tube & 200 & 3 & $1.5 * *$ & 5.3 & 7.1 & $\mathbf{1 0 6 0 . 3}$ \\
\hline \multirow{2}{*}{4} & $\begin{array}{l}\text { (Ke) - Sensor by Kesavan et al. [9] } \\
\text { (steel rod with a silicone rubber } \\
\text { coating) }\end{array}$ & $\begin{array}{c}\text { Steel rod } \\
\text { Silicone rubber }\end{array}$ & 200 & 5 & 0 & 19.6 & & 3927.0 \\
\cline { 2 - 9 } & Overall & 42.1 & 11 & & & 95.0 & $\mathbf{4 0 0 2 . 4}$ \\
\hline
\end{tabular}

*not real sensor, included for study purposes; **not provided by authors, assumed based on market search.

Figure 4 shows the geometrical properties of the original sensors and those of the equivalent sensor used in the FE analysis. 
Influence of mechanical and geometrical properties of embedded long-gauge strain sensors to the accuracy of the strain measurement

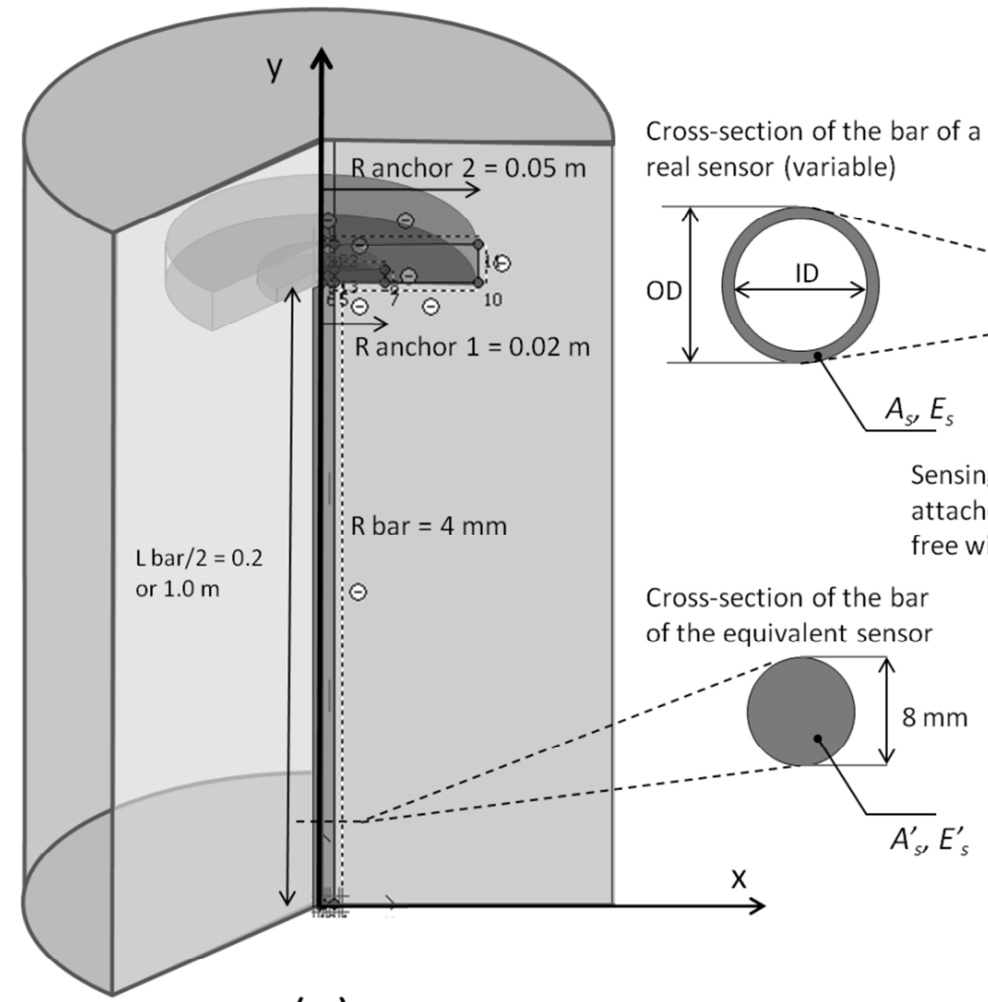

(a)
Cross-section of the bar of a or (variable)

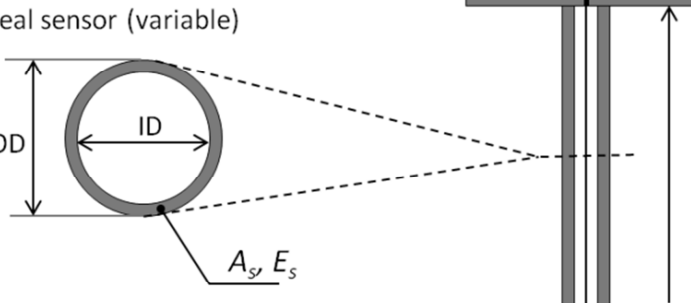

Sensing element, $\quad \mathrm{L}$ bar $=0.4$ attached to the ends, $\quad$ or $2.0 \mathrm{~m}$ ree within the bar

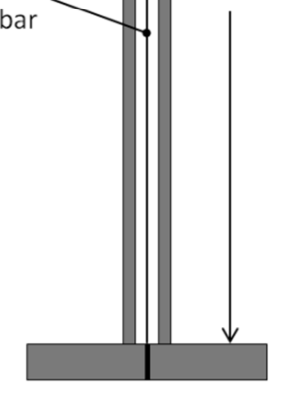

(b)

Figure 4. Geometry of the sensors considered in the parametric study. (a) Geometrical properties of the equivalent sensor used in 2D FE analysis. (b) Basic geometry of the original sensors.

Since the dimension of the cross-section of the bar of the equivalent sensor was kept constant to simplify analysis, the mechanical properties of the bar model had to be modified, in order to for model to obtain similar mechanical properties as the real sensors. The study demonstrated that two embedded sensors with different diameters cannot be assumed equivalent if they have the same axial stiffness only (i.e., the product of Young modulus and the area of the cross section "EA"), but the mechanical properties of the host material and the geometrical properties of the modeled and real sensors have to be taken into account. If the diameter of the bar of the real sensor is different than the diameter of the bar of the modeled sensor, then the contribution of the host material that fills the space difference between the real and modeled equivalent sensor has to be accounted in overall stiffness of modeled sensor.

Let $E_{s}, A_{s}$, and $A_{O}$ be the Young modulus, the area of the cross-section, and the outer area of the bar of the real sensor respectively $\left(A_{s}=\pi\left(O D^{2}-I D^{2}\right) / 4, A_{O}=\pi O D^{2} / 4\right.$, where $O D$ and $I D$ are outer and inner diameters of the bar); let $E_{m}$ be the Young Modulus of the host material and let the bar of the modeled sensor, which is to be considered as equivalent to a real sensor has diameter of $8 \mathrm{~mm}$ and the area of the cross-section $A^{\prime}{ }_{s}=\pi 8^{2} / 4=50.3 \mathrm{~mm}^{2}$; then the equivalent Young modulus $E^{\prime}{ }_{s}$ is expressed by Equation (5): 
Influence of mechanical and geometrical properties of embedded long-gauge strain sensors to the accuracy of the strain measurement

$$
E_{s}^{\prime}=\frac{E_{s} A_{s}+E_{m}\left(A_{s}^{\prime}-A_{O}\right)}{A_{s}^{\prime}}
$$

2

3

Equation (5) demonstrates that the properties of the host material need also to be taken into account when converting real sensor into equivalent sensor. Table 3 shows the Young modulus of the equivalent sensors with bar diameter of $8 \mathrm{~mm}\left(A_{s}^{\prime}=50.3 \mathrm{~mm}^{2}\right)$. Properties of host materials a-e quoted in Table 3 are presented in Table 1.

Table 3. Equivalent Young moduli for $8 \mathrm{~mm}$ diameter sensor bars in different host materials.

\begin{tabular}{|c|c|c|c|c|c|c|}
\hline \multicolumn{2}{|r|}{ SENSOR } & \multicolumn{5}{|c|}{$\mathbf{E}_{\mathrm{s}}^{\prime}\left[\mathrm{kN} / \mathrm{mm}^{2}\right]$, HOST MATERIAL } \\
\hline Id. & Description & $\mathbf{a}$ & b & c & d & e \\
\hline 1 & (GI) - SMARTEC sensor (Glisic [10]) & \multicolumn{5}{|c|}{0.44} \\
\hline 2 & (In) - Intermediate sensor* & \multicolumn{5}{|c|}{10.04} \\
\hline 3 & (Le) - Sensor by Leng et al. [19] & 21.24 & 22.21 & 27.97 & 40.17 & 44.58 \\
\hline 4 & (Ke) - Sensor by Kesavan et al. [9] & 79.47 & 78.47 & 72.50 & 59.85 & 55.28 \\
\hline
\end{tabular}

\subsection{Modeling of the Interface between Different Materials}

The interaction between the host material and the sensors was modeled by using special "interface" elements with Möhr-Coulomb's constitutive model. Different properties of the interface elements were set for different sensor components. The study has shown that the deformation is practically completely transmitted from the host material to sensors over the anchor pieces, i.e. only a negligible part of the deformation is transmitted through the interface between the sensor bar and the host material. Thus, a "non-frictional" interface elements were used to model interaction between the bar and the host material, i.e., the interaction between the bar and the host material was neglected. The interaction between the anchor piece and the host material is stronger and two sets of properties of the interface elements were studied:

- Rough interface: the interaction between the anchor piece and the host material was assumed to be very good; a strength reduction factor of 0.5 was set to represent this "rough" interface (the reduction factor relates the interface strength, which includes friction and adhesion, to the host material strength that includes friction angle and cohesion; properties of the host material are given in Table 1). A reduction factor of 0.5, typical in the analysis of wall-soil interfaces (see [14] and [18]), has been chosen. Nevertheless, previous sensibility analysis have shown that increasing the interface strength reduction factor over 0.5 , and up to 1.0 , has a negligible influence on the results.

- Smooth interface: the interaction between the anchor piece and the host material is significant, but not as good as in the case of a rough interface; a cohesion of $0 \mathrm{MPa}$ and a friction angle of $11.5^{\circ}$ were used; these values describe, for example, a smooth-steel - concrete interface, as per Calderón et al. [20], Adam et al., [21,22,23] or Johansson and Gylltoft [24,25]. 
Influence of mechanical and geometrical properties of embedded long-gauge strain sensors to the accuracy of the strain measurement

1 The general FE model was first validated (see appendix A) and then used in the parametric study (Section

2 4). The validation of the model is crucial, but it does not represent the main topic of the paper.

3 Consequently, in order to simplify organization of the paper and emphasize the main results, it is moved

4 in Appendix A, while the parametric study (which encompasses the main topic of the paper) is presented

5 in the next section.

6

7

\section{Parametric Study}

It was demonstrated in Appendix A that the mechanical behavior of embedded long-gauge sensors and surrounding host material can be accurately reproduced by the developed FE models. A parametric study presented in this section uses developed FE model to generate results for a wide range of possible sensor and host material properties, with aim to understand how variations in these properties would influence the accuracy of the measurement performed by the modeled sensors if embedded in modeled host material. In order to evaluate the accuracy of a given long-gauge sensor, the relative difference (or relative error) was defined as follows:

$$
\delta \varepsilon[\%]=\frac{\varepsilon_{s}-\varepsilon_{v}}{\varepsilon_{v}} \cdot 100
$$

where $\varepsilon_{v}$ is modeled non-perturbed strain in host material, i.e., the one that would take place if the sensor did not exist, and $\varepsilon_{s}$ is the modeled strain measured by the sensor, i.e. the one that would take place if the sensor is embedded in the host material.

Both types of strain were calculated for different load levels, ranging from zero to the unconfined compression strength of the host material $\left(f^{\prime}{ }_{c 0}\right)$. In order to highlight comparison between different materials a "characteristic error" was defined as the one that takes place at a load equal to $0.75 \cdot f^{\prime} c$.

\subsection{Studied Parameters and Cases}

The following variables were considered in the parametric study:

- Host materials: five different host materials were analyzed with Young moduli ranged between 0.2 and $27.3 \mathrm{GPa}$; the materials' properties are presented in Table 1 ; to simplify presentation, the materials are briefly named $a, b, c, d$, and $e$, with " $a$ " being the softest and " $e$ " being the stiffest;

- Sensors: four types of sensor materials were simulated; their properties are given in Tables 2 and 3 and Figure 4; equivalent Young moduli for the sensors ranges from 0.44 to $79.47 \mathrm{GPa}$; for simpler presentation the sensors are named $G l, I n, L e$, and $K e$, as shown in Table 2, with "Gl" being the softest and " $K e$ " being the stiffest; the following geometrical properties of the sensor were used:

- Gauge length: two lengths were analyzed: $0.4 \mathrm{~m}$ and $2.0 \mathrm{~m}$ (half-lengths of $0.2 \mathrm{~m}$ and $1.0 \mathrm{~m}$ );

- Radius of the anchor piece: two radii were analyzed: $0.02 \mathrm{~m}$ and $0.05 \mathrm{~m}$ (see Figure 4); 
Influence of mechanical and geometrical properties of embedded long-gauge strain sensors to the accuracy of the strain measurement

- Interface between the anchor and the host material: two types of interface were analyzed: " $i$ " corresponds to the "rough" interface, while "ii" corresponds to the "smooth" interface (see Section 3); the interface between the sensor bar and the host material was considered as "non-frictional".

The above cases include a wide range of parameters that simulate the most common materials found in civil and geotechnical engineering, and the most common sensor types applied in practice and allow for a comprehensive parametric study. A total of 136 FEM simulations were carried out.

\subsection{The Results of FE Analysis}

Figures 5 and 6 show typical outcomes of the FE analysis. Figure 5a compares the displacement field in the vicinity of two sensors with identical interface " $i$ ", host material " $b$ ", sensor type "Ke", and gauge half-length " 0.2 " (full length of $0.4 \mathrm{~m}$ ), but different radii of anchor piece $(0.02 \mathrm{~m}$ and $0.05 \mathrm{~m})$. The image on the left is mirrored to highlight the difference from the image on the right: the displacement field in host material is more perturbed by the sensor with a smaller radius of the anchor piece.

Figure 5b shows the relative shear field (ratio between deviatoric stress and shear strength) for the presented sensors. Again, image on the left is mirrored. The shear stress field in host material is more perturbed by the sensor with a smaller radius of the anchor piece.

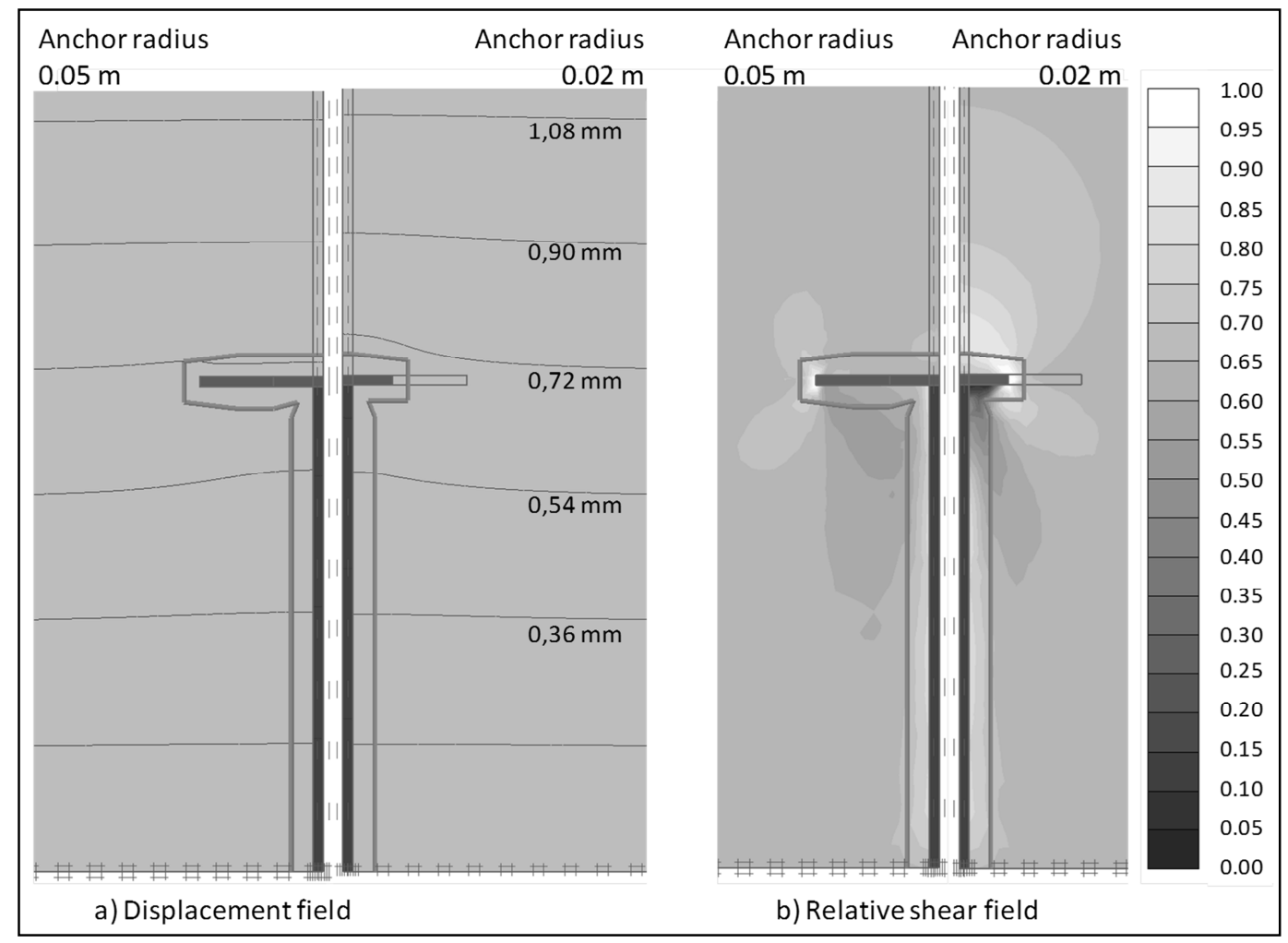

Figure 5. a) Displacement fields and b) relative shear fields (ratio between deviatoric stress and shear strength) for cases "i/b/0.2/Ke/0.05" (left image) and "i/b/0.2/Ke/0.02" (right image). 
Influence of mechanical and geometrical properties of embedded long-gauge strain sensors to the accuracy of the strain measurement

1

2 Figure 6 exemplifies the stress-strain curves obtained by FEM simulation for host material " $a$ ", sensor 3 half-lengths of $0.2 \mathrm{~m}$ (full length of $0.4 \mathrm{~m}$ ), and rough anchor-host material interface " $i$ " subjected to the full range of loads. The curves show the differences generated by varying the stiffness of the sensor and the size of the anchor pieces. The results in Figure 6 show that the relative error is by absolute value larger for stiffer sensors, and in the case of the sensors with the same stiffness the relative error is by absolute value larger for sensor with smaller radius of anchor piece. For very stiff sensors with small anchor pieces a non-linear stress-strain relation is noticed (e.g. see cases "i/a/0.2/Ke/0.02" or "i/a/0.2/Le/0.02"), thus the relative error increase by absolute value for higher load levels.

To simplify evaluation, a "characteristic error" was defined as the relative error at the load of $0.75 \cdot f$ ' $c 0$. Figures 7-9 show the characteristic error as a function of Young modulus of the host material.

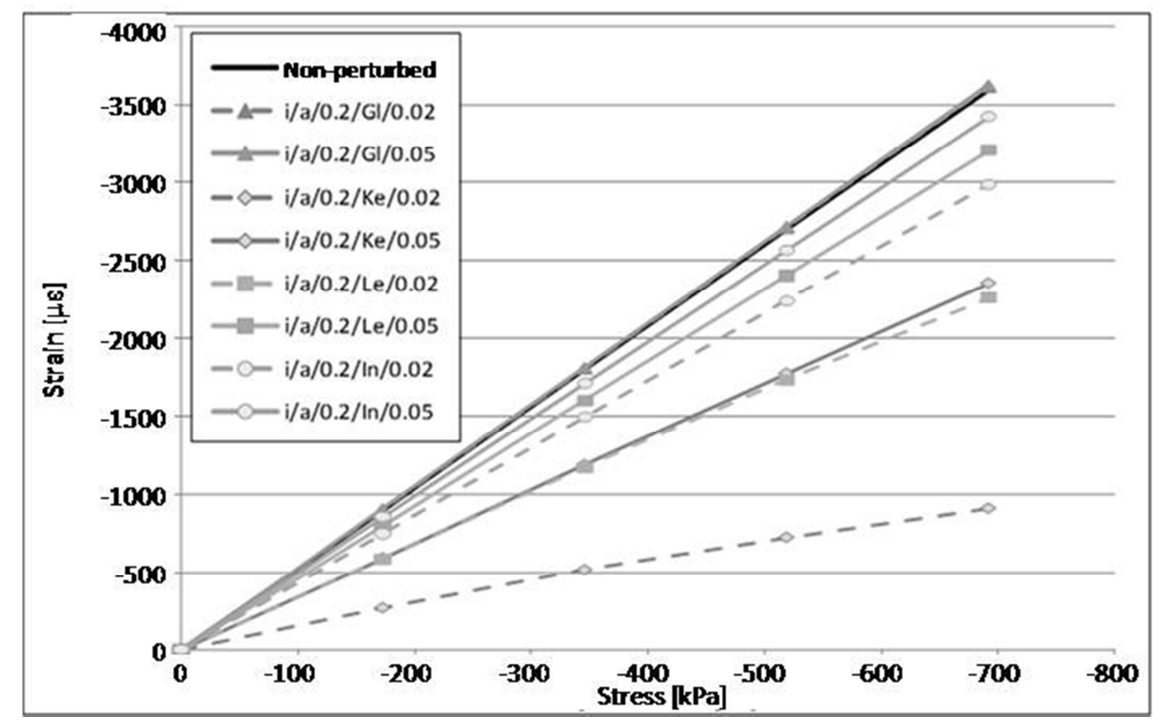

Figure 6. Stress-strain curves of the sensors for an " $a$ " host material, $0.4 \mathrm{~m}$ long sensors $(0.2 \times 2 \mathrm{~m})$ and rough anchor-host material interface $(i)$

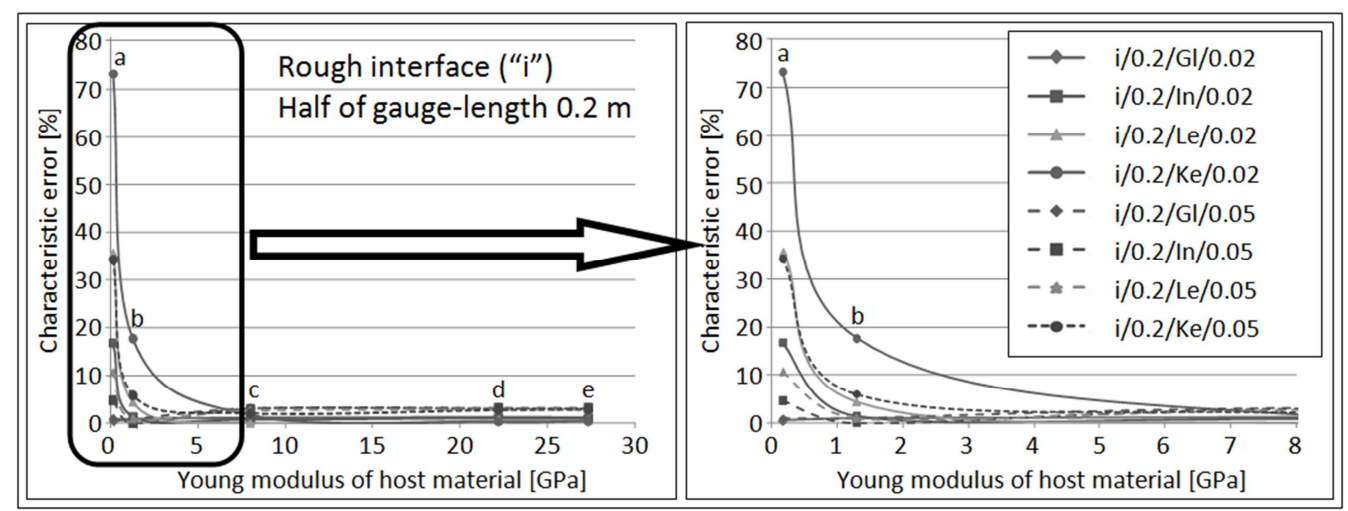


Influence of mechanical and geometrical properties of embedded long-gauge strain sensors to the accuracy of the strain measurement

1 Figure 7. Characteristic error for $0.4 \mathrm{~m}$-long sensor with rough anchor-host material interface; left: full range of host material Young moduli; right: zoom to lower range of Young's moduli.

Figures 7 and 8 show that for the rough interface, in general, the characteristic error rapidly decreases as the Young modulus of host material increases. For example, in materials $c, d$, and $e$, the characteristic error is small, and the sensor properties do not influence significantly the characteristic error. However, the characteristic error in soft materials (e.g. materials $a$ and $b$ ) strongly depends on the sensor properties, and softer sensors with larger anchor pieces and longer gauge lengths generate smaller errors.

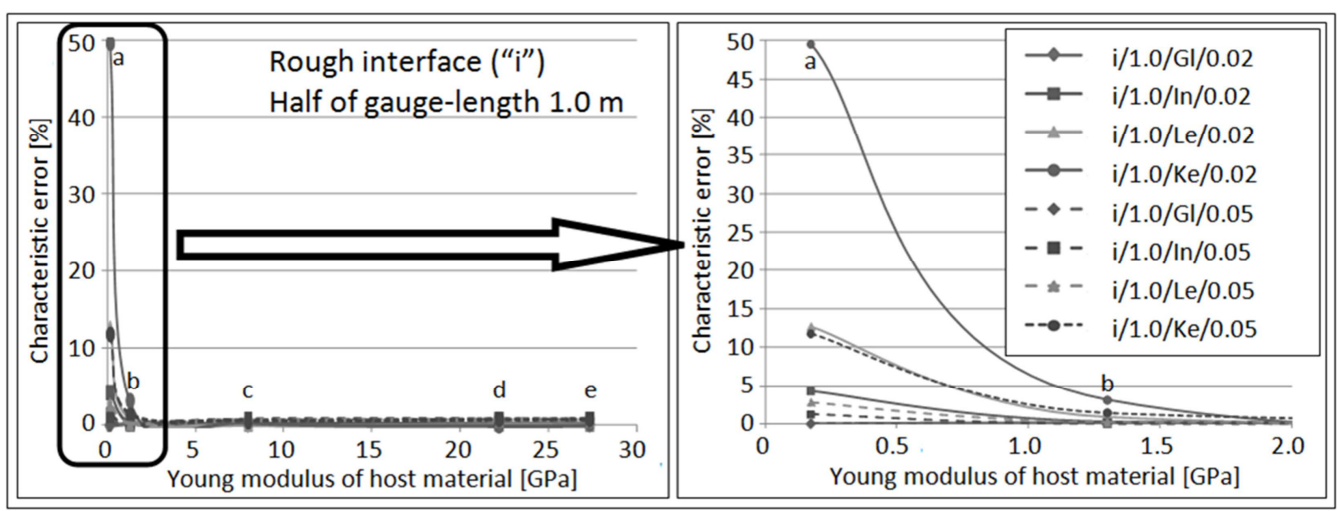

Figure 8. Characteristic error for $2.0 \mathrm{~m}$-long sensor with rough anchor-host material interface; left: full range of host material Young moduli; right: zoom to lower range of Young's moduli.

Figure 9 shows that for the smooth interface, in general, the characteristic error strongly depends on the sensor properties. Stiffer sensors (e.g. " $K e$ " and " $L e$ ", but also "In", depending on geometrical properties) with smaller anchor pieces and shorter gauge lengths generate large characteristic errors regardless the stiffness of the host material. Nevertheless, softer sensors (e.g. " $G l$ " and to certain extent "In") with larger anchor pieces and longer gauge lengths can provide with more accurate measurements. Detailed analysis of all results is presented in the next section.

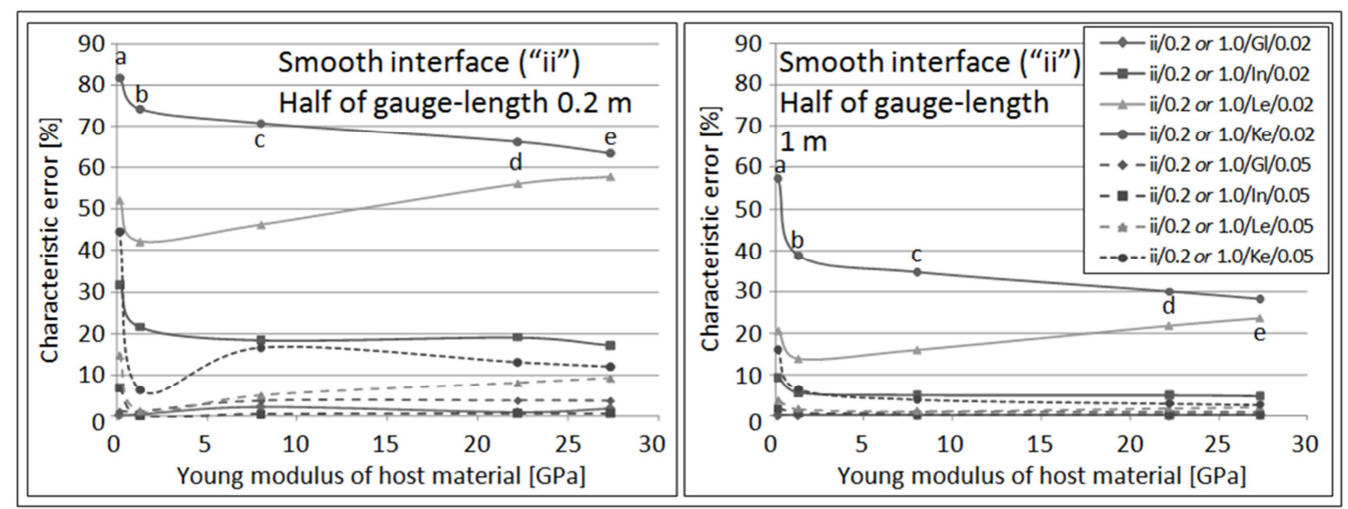


Influence of mechanical and geometrical properties of embedded long-gauge strain sensors to the accuracy of the strain measurement

Figure 9. Characteristic error for a smooth anchor-host material interface; left: sensor gauge-length equal to $0.4 \mathrm{~m}$; right: sensor gauge-length equal to $2.0 \mathrm{~m}$.

\subsection{Analysis of Results and Recommendations}

The results presented in previous sub-section clearly show the importance of the long-gauge sensor and host material properties, and their influence to the accuracy of the strain measurement. To achieve an accurate strain measurement in a given host material using embedded long-gauge sensor, the geometrical and mechanical properties of the sensor have to be carefully chosen. The most important findings of this study and the consequent recommendations for the users of long-gauge sensors are given as follows:

- Host material: in general for all the sensors modeled in this study, the higher stiffness of the host material had as a consequence lower error in the simulated strain measurement. This trend is shown in Figures 7 and 8; the only exception is sensor " $L e$ " when a smooth anchor-host material interface is considered; the main reason for this behavior is the fact that at the equivalent elastic modulus of the sensor " $L e$ " depends on the host material properties (see Equation 5), and the stiffness of the equivalent sensor increases with increase of the stiffness of the host material (see Table 3); the sensor "Ke" has a "changing" equivalent modulus of elasticity too, but its value decreases with increase of the stiffness of the host material; in addition the change is not as accentuated as in the case of the sensor " $L e$ ".

- Sensor equivalent stiffness: the sensors with a very low equivalent stiffness $\left(E_{s}{ }_{s} A^{\prime}<22 \mathrm{kN}\right)$, such as the sensor " $G l$ ", has an excellent accuracy, and relative error is by absolute value smaller than $4 \%$ regardless the other geometrical and mechanical properties; other geometrical and mechanical properties, if appropriately chosen, can significantly improve the accuracy of the strain measurement; in fact the sensors that feature higher stiffness need to have the other geometrical and mechanical properties suitably selected in order to achieve acceptable accuracy of the measurement.

- Interaction between the anchor piece and the host material: quality of interaction between the anchor piece and the host material has the largest influence to the accuracy of the measurement as it controls the goodness of the strain transfer from the host material to the sensor; a weak interaction, i.e. smooth interface ( "ii"), has as a consequence very large errors in the strain measurements for sensors which stiffness is medium to high, such as " $L e$ " or "Ke"; these two sensors practically should not be used if their anchor pieces have low interaction with the host material, unless their length is longer than $2 \mathrm{~m}$, and anchor pieces have radius of $0.05 \mathrm{~m}$; even then, the relative error is by absolute value not smaller 
Influence of mechanical and geometrical properties of embedded long-gauge strain sensors to the accuracy of the strain measurement

than 2 to 5\%; the increase of interaction between the anchor piece and the host material can improve the accuracy of the strain measurements several times; it is therefore recommended to use exclusively sensors with anchor pieces that strongly interact with the host material; interaction can be improved by indenting the lateral areas of the anchor pieces and by increasing their overall roughness; if a good interaction is achieved, then practically all the sensors except the sensor "Ke" can be used in host material with Young modulus higher than $3 \mathrm{GPa}$, and the " $K e$ " sensor can be used for host material with Young modulus higher than $8 \mathrm{GPa}$ for gauge lengths not shorter than $0.4 \mathrm{~m}$.

- Radius of the anchor piece: has also important influence, especially for stiffer sensors such as " $K e$ " or " $L e$ "; for these sensors the increase of radius of the anchor piece from $0.02 \mathrm{~m}$ to $0.05 \mathrm{~m}$ improves the accuracy more than two times; while this statement is valid for "smooth" anchor pieces regardless the stiffness of the host material, for "rough" anchor pieces this statement is valid only for host material with Young modulus smaller than $3 \mathrm{GPa}$ approximately; "rough" anchor pieces with large radius perturb too much the strain field in their surrounding which causes increase in the error of the measurement; thus if the interaction between the anchor piece and the host material is good, then larger anchor pieces are recommended only if the stiffness of the host material is less than $3 \mathrm{GPa}$ approximately; however, if the interaction is weak, then the increase of the radius of anchor pieces is in general recommended.

- Gauge length: as the strain and displacement fields are the most perturbed in the areas surrounding the anchor pieces, the error of the strain measurement decreases with the increase of the gauge length, because the error due to perturbation is distributed over longer length; in the case of "rough" anchor pieces the relative error is by absolute value smaller than $5 \%$ for all sensors (regardless the size of the anchor pieces) if the host material stiffness is higher than $1.5 \mathrm{GPa}$, while for the $0.4 \mathrm{~m}$ long sensor the same is valid only if the material stiffness is higher than $5 \mathrm{GPa}$; thus for softer host materials, besides an increase in anchor pieces, it is recommended to use sensors with larger gauge lengths.

The results revealed that the quality of interaction between the anchor piece and the host material (i.e., the "roughness" of the anchor interface) has the biggest influence to the accuracy of the strain measurement. The second most important parameter that influences the accuracy of the strain measurement is the ratio between the equivalent Young modulus of sensor and the Young modulus of host material. This statement was further verified by observing the dependence of the characteristic error on the ratio of two moduli.

Figure 10 left shows that for a given sensor geometry (gauge lengths and the anchor piece size), there is parabolic correlation between the $E_{s} / E_{m}$ ratio and the characteristic error (note that the $x$-axis in the figure is in logarithmic scale), having provided that the anchor-host material interface is "rough".

However, Figure 10 right shows that in the case of a "smooth" interface, the characteristic error is big for $E_{s}{ }^{\prime} / E_{m}>0.1$, but an explicit dependence rule cannot be established due to large scatter of the results. This 
Influence of mechanical and geometrical properties of embedded long-gauge strain sensors to the accuracy of the strain measurement

1 confirms that for a "smooth" interface the behavior of the sensor is dependent not only on the geometry 2 and the elastic properties of the sensors and the host material, but also on some other factors. Among performance of the sensors for $E^{\prime}{ }_{s} / E_{m}$ ratios over 0.1 .

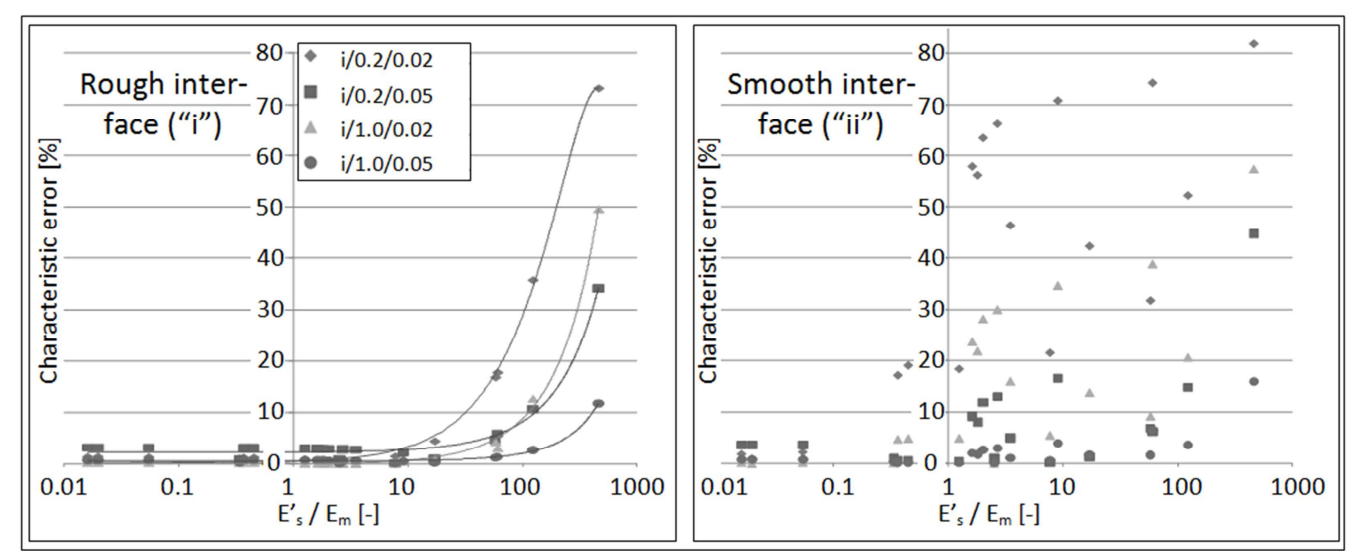

Figure 10. Characteristic error vs. ratio between equivalent Young modulus of sensor $E_{s}$, and Young modulus of host material $E_{m}$; left: for rough anchor-host material interface "i"; right: for a smooth anchorhost material interface "ii".

\section{Conclusions}

A parametric study based on FE analysis has been carried out in order to study the influence of geometrical and mechanical properties to the accuracy of measurement of embedded long-gauge sensors. Involved materials were assumed to have the Möhr-Coulomb elastic, perfectly plastic behavior. A suitability of the FE model for the analysis was verified using the experimental results of two cases reported in the literature and one on-site application in which three sensors with different gauge lengths were embedded in the concrete (see Appendix A). The study revealed the influence of geometrical and mechanical properties of the host material and the sensor to the accuracy of the strain measurement.

The goodness of the interaction between the anchor piece and the host material (roughness of the anchor piece) was found to be the most influential property: accurate measurement necessities sensors with anchor pieces that have a very good interaction with the host material. (i.e., "rough" anchor pieces). The ratio between the equivalent Young modulus of sensor and the Young modulus of the host material also have strong influence to the accuracy of the measurement, and lower ratios provide with more accurate measurement. Longer gauge lengths provide with more accurate measurements, since the error generated in the proximity of the anchor piece is distributed over longer measurement basis. Finally, the role of the radius of anchor piece is also important: for smooth interfaces bigger radius provides with more accurate 
Influence of mechanical and geometrical properties of embedded long-gauge strain sensors to the accuracy of the strain measurement

measurement, while in the case of the rough interfaces bigger radius provides improvements only if the sensor is embedded in a very soft host material. The following recommendations result from this study:

- To use sensors which anchor pieces provide with good interaction with the host material; lateral surfaces of the anchor pieces should be indented and overall surface made rough;

- To use sensor with low ratio between the equivalent Young modulus of sensor and the Young modulus of the host material $\left(E_{s}, E_{m}\right)$;

- If the ratio between the equivalent Young modulus of sensor and Young modulus of the host material $\left(E_{s}, E_{m}\right)$ is high, then bigger anchor pieces are recommended;

- To use sensors with long gauge length, however, excessive gauge length can introduce other types of errors in structures and soils where the strain field significantly varies along the gauge length [13].

For most sensor configurations a gauge factor can be adjusted in order to take into account and compensate for the strain transfer error, having provided that geometrical and mechanical characteristics of the sensor and the host material are known. However, this is not applicable for those configurations that lead to significant error since for them the relationship between real and measured strain is not linear, especially at high stress levels (see Figure 6).

\section{Acknowledgments}

The authors would like to thank to the Spanish Ministry of Education, which support received under the National Program for Mobility of Researchers (O.M. EDU/1456/2010, ref. PR2010-0293) enabled the joint work that made possible this study. The Streicker Bridge project was realized with help of Turner Construction Co., HNTB, A.G. Construction Corp., Vollers Excavating \& Constr., SMARTEC SA, Micron Optics, Princeton Facilities, and staff and students of CEE department of Princeton University.

\section{References}

1. Hornby, I.W. (1992). The Vibrating Wire Strain Gage, in ed. Window, A.L. Strain gauge technology, pp325-346, Elsevier science publishers, Ltd., Barking, UK.

2. Measures, R. (2001). Structural monitoring with fiber optic technology, p717, Academic Press, London 3. Feng, X., Sun, C., Zhang, X. and Ansari, F. (2010). Determination of the coefficient of thermal expansion with embedded long-gauge fiber optic sensors, Meas. Sci. Technol. 21: 065302 (8pp).

4. Rodrigues, C. and Inaudi, D. (2010). Laboratory and Field Comparison of Long-gauge Strain Sensing Technologies, European Workshop on Structural Health Monitoring, Paper on CD, Sorrento Italy.

5. Glisic, B. and Inaudi, D. (2007). Fibre Optic Methods for Structural Health Monitoring, John Wiley \& Sons, Inc., p262, Chichester, UK. 
Influence of mechanical and geometrical properties of embedded long-gauge strain sensors to the accuracy of the strain measurement

1 6. Ansari, F. (2007). Practical Implementation of Optical Fiber Sensors in Civil Structural Health 2 Monitoring, Journal of Intelligent Material Systems and Structures, 18(8): 879-889.

3 7. Li, H.N., Zhou, G.D., Ren, L. and Li D.S. (2009). Strain Transfer Coefficient Analyses for Embedded 4 Fiber Bragg Grating Sensors in Different Host Materials, J. Eng. Mech. 135:12(1343) (11 pages). 8. Torres, B., Payá-Zaforteza, I., Calderón, P.A. and Adam, J.M. (2011). Analysis of the strain transfer in a new FBG sensor for Structural Health Monitoring, Engineering Structures, 33:539-548. 9. Kesavan, K., Ravisankar, K., Parivallal, S., Sreeshylam, P. and Sridhar, S. (2010). Experimental studies on fiber optic sensors embedded in concrete, Measurement, 43:157-163. 10. Glisic, B. (2000). Fiber optic sensors and behavior in concrete at early age, Ph.D. thesis, no. 2186, Ècole Polytechnique Féderal de Lausanne, p146, Lausanne, Switzerland. 11. Azenha, M., Faria, R., Ferreira, D. (2009). Identification of early-age concrete temperatures and strains: Monitoring and numerical simulation, Cement \& Concrete Composites 31:369-378. 12. Bhatt, P., MacGinley, T.J. and Choo, B.S. (2006). Reinforced concrete: design theory and examples 3rd ed. p767, Taylor \& Francis, Abingdon, UK.

13. Glisic, B. (2011). Influence of the gauge length on the accuracy of long-gauge sensors employed in monitoring of prismatic beams, Meas. Sci. Technol. 22 (3):035206 (13pp). 14. Plaxis 2D (2002). Reference Manual. Version 8.2. PLAXIS B.V. 15. Malvern, L.E. (1977). Introduction to the Mechanics of a Continuous Medium, Prentice-Hall, Inc. 16. Timoshenko S., Young, D.H. (1945). Theory of Structures, McGraw-Hill Book Company, Inc. 17. CEB-FIB Model Code 90. (1991). Lausanne. 18. Departments of the Army and Air Force, USA (1983). Technical Manual TM 5-818-1/AFM 88-3, Chapter 7, Soils and Geology Procedures for Foundation Design of Buildings and Other Structures. 19. Leng, J.S., Winter, D., Barnes, R.A., Mays, G.C. and Fernando, G.F. (2006). Structural health monitoring of concrete cylinders using protected fiber optic sensors, Smart Mater. Struct., 15: 302-308. 20. Calderón, P.A., Adam, J.M., Ivorra, S., Pallarés, F.J. and Giménez, E. (2009). Design strength of axially loaded RC columns strengthened by steel caging, Materials and design, 30:4069-4080. 21. Adam, J.M., Ivorra, S., Pallarés, F.J., Giménez, E. and Calderón, P.A. (2009). Axially loaded RC columns strengthened by steel caging finite element modelling. Constr Build Mater; 20(6):2265-76. 22. Adam, J.M., Ivorra, S., Pallarés, F.J., Giménez, E. and Calderón, P.A. (2008). Column-joint assembly in RC columns strengthened by steel caging. Proc. ICE - Struct Build;161(6):337-48. 23. Adam, J.M., Ivorra, S., Pallarés, F.J., Giménez, E. and Calderón, P.A. (2009). Axially loaded RC columns strengthened by steel caging. Proc. ICE - Struct Build;162(3): 199-208. composite columns under various means of load application, Steel Compos Struct, 4:393-410. 
Influence of mechanical and geometrical properties of embedded long-gauge strain sensors to the accuracy of the strain measurement

1 25. Johansson, M. and Gylltoft, K. (2002). Mechanical behaviour or circular steel-concrete composite 2 stub columns, J Struct Eng, 128(8):1073-81.

3 26. Glisic, B. (2011). Streicker Bridge: an on-site SHM laboratory at Princeton University campus, Proc.

4 of SMAR, Paper No. 306, Dubai, UAE.

5 27. U.S. Army Corps of Engineers, CECW-EG (1990). Engineering and Design-SETTLEMENT 6 ANALYSIS, Engineer Manual 1110-1-1904. 
Influence of mechanical and geometrical properties of embedded long-gauge strain sensors to the accuracy of the strain measurement

\section{APPENDIX A. Validation of Numerical Model}

The aim of the work described in this appendix is to verify that the FE model developed in Section 3 represent the behavior of a system consisting of an embedded long-gauge sensor and the host material. The validation of the model is carried out using the data from two laboratory tests and a real on-site application. The data of laboratory tests was reported in literature [9] and [19]. In these tests the host material was hardened concrete, but the stiffness of the sensor bar was different, as well as the dimensions of the anchor pieces. The on-site application provides with the measurements of three sensors with different gauge lengths embedded in non-hardened concrete [13].

\section{A.1 The First Laboratory Test (Kesavan et al. [9])}

Kesavan et al. [9] report the results of a $60 \mathrm{~mm}$ long embedded sensor consisting of a $5 \mathrm{~mm}$-diameter steel rod with sensing optical fiber, covered with a $3 \mathrm{~mm}$-thick silicone rubber coating. At both ends the sensor has anchor pieces in form of $4 \mathrm{~mm}$-thick steel disc with diameter of $25 \mathrm{~mm}$, attached to the rod by a nut. The sensors were embedded in $300 \mathrm{~mm}$ long concrete cylinders with diameter of $150 \mathrm{~mm}$, and tested under uniaxial compression load that reached $200 \mathrm{kN}$ (11.3 MPa). The strain measured by the embedded sensor was compared to measurements of the four electrical strain gauges placed at the surface of the concrete cylinders. The FE model was set as follows:

- A vertical outer boundary and the upper boundary were set free in both directions; boundary at the bottom was set free only in horizontal direction (constraints were imposed in the vertical direction);

- Due to geometrical irregularities existing at the top surface of the nut, a "rough" interface element is set at the contact between this upper surface and the concrete, while the "smooth" interface element is used at the contact between the anchor piece and the concrete; the interface between the bar and the concrete was kept "non-frictional” (see Section 3);

- Geometrical and mechanical properties of the concrete and the sensor are taken from [9] and presented in Tables 1-3; the properties of interface elements are given in Section 3.3.

Figure A.1 shows the mesh, geometrical properties, and interface properties used in the FE model, along with the comparison between the FE model and the test results. The relative difference between FE model and measurements was for the embedded sensor less than 1\%; thus comparison demonstrated that the FE model reproduced well the experimental results and validated the FE model. 
Influence of mechanical and geometrical properties of embedded long-gauge strain sensors to the accuracy of the strain measurement

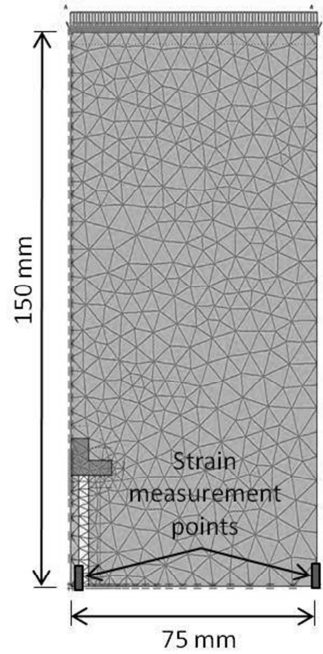

(a)

(b)

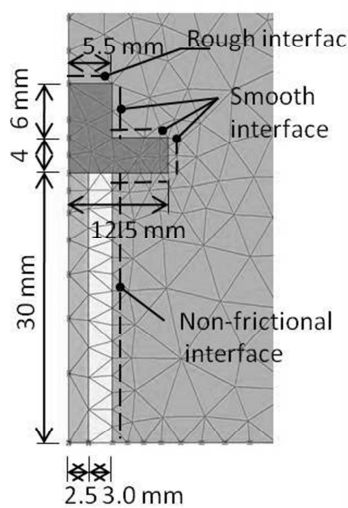

(c)

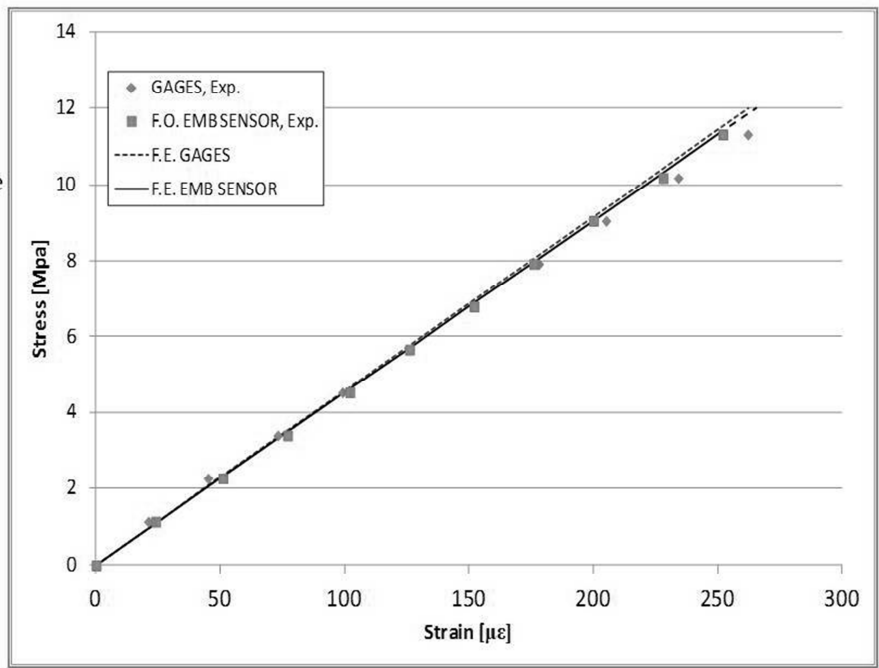

Figure A.1. a) FE model of the sensors reported in [7], b) FE model - detail, c) test results vs. FE model.

\section{A.2 The Second Laboratory Test (Leng et al. [19])}

Leng et al. [19] reported the results of an $80 \mathrm{~mm}$-long embedded sensor consisting of a steel tube with OD of $3 \mathrm{~mm}$ and the anchor pieces in form of $5 \mathrm{~mm}$-thick steel discs with diameter of $10 \mathrm{~mm}$. The lateral surfaces of the discs are indented in order to have better interaction with the host material. As the paper does not report on the thickness of the steel tube, a market search was performed to find the most common available dimension, and as a result a tube with an ID of $1.5 \mathrm{~mm}$ was found and used in the FE model. Sensitivity study has shown that small variation of the ID does not influence the results of the validation because it does not affect significantly the equivalent Young modulus of the sensor.

The fiber optic sensing element was attached at ends of the steel tube. The strains are then obtained by dividing the relative displacement between the two ends of the sensor by the original distance between them. The sensors were embedded in $200 \mathrm{~mm}$-long concrete cylinders with diameter of $100 \mathrm{~mm}$, and tested under compression loads up to $12.7 \mathrm{MPa}$. The strains measured by the embedded sensor were compared to those measured by electrical strain gauges placed at the surface of the concrete cylinders. The sensor had a steel tube extension at the lower anchor piece that reached the bottom of the concrete specimen. A thick silicone-rubber protection for the fiber optic cable was placed beyond the upper anchor piece of the sensor. Consequently, the sensor was not symmetric with respect to the horizontal plane and only axial symmetry was taken into account in the FE model (sees Figure A.2). The FE model was set as follows:

- A vertical outer boundary was set free in both directions; rigid beam linear elements were set at upper and lower boundary to simulate the steel plates of the testing equipment; 
Influence of mechanical and geometrical properties of embedded long-gauge strain sensors to the accuracy of the strain measurement

- A "rough interface" is set at the contact between the upper surface of the nut and the concrete, while "smooth" is used at the contact between the anchor piece and the concrete (see Section 3); the interface between the bar and the concrete was kept "non-frictional" (see Section 3);

- Geometrical and mechanical properties of the concrete and the sensor are taken from [19] and presented in Tables 1-3; the properties of interface elements are given in Section 3.3.

Figure A.2 shows the mesh, geometrical properties, and interface properties used in the FE model, along with comparison between the FE model and the test results. The relative difference was less than $5 \%$ for medium and high strain values (>100 $\mu \varepsilon$ ), and less than $10 \%$ in general. Thus comparison again demonstrated that the FE model reproduced well the experimental results and validated the FE model.

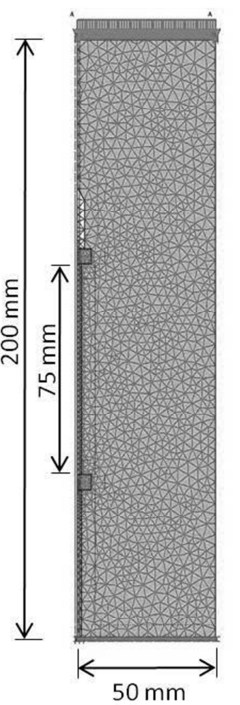

a)

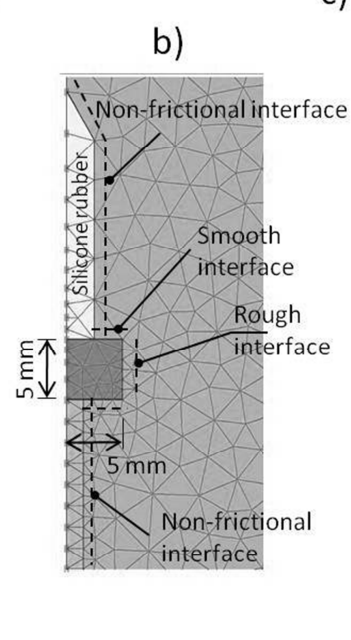

c)

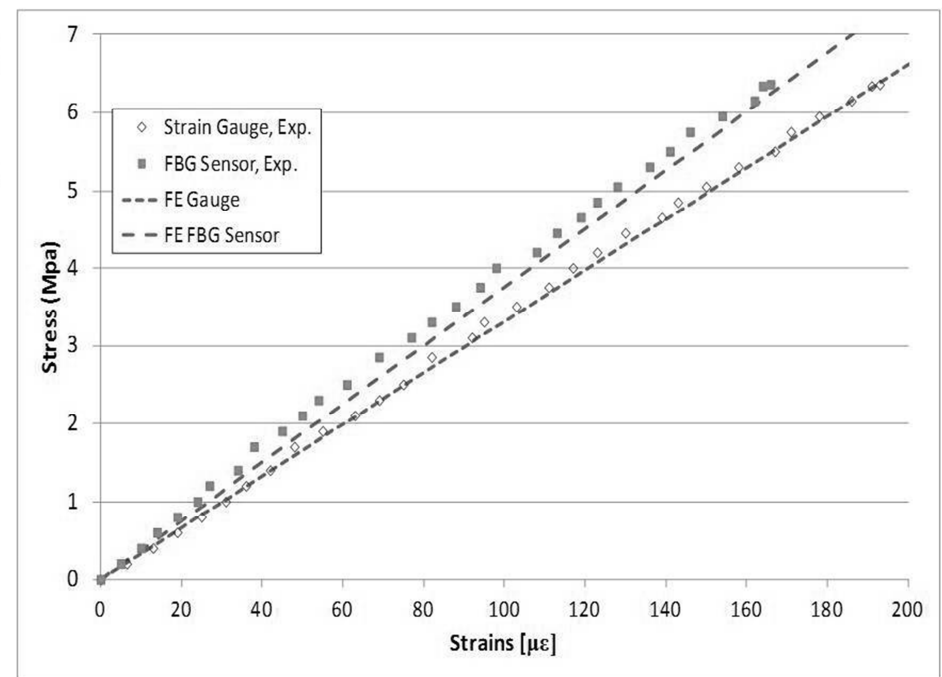

Figure A.2. a) FE model of the sensors reported in [2], b) FE model - detail, c) test results vs. FE model.

\subsection{The On-site Tests}

The "south-east leg" (SE-leg) of the Streicker Bridge at Princeton University campus is a curved continuous girder, made of post-tensioned concrete and supported by weathering steel columns [26]. The bridge has been equipped with long-gauge fiber-optic sensors with absolute measurement error estimated to $4 \mu \varepsilon\left(4 \cdot 10^{-6} \mathrm{~m} / \mathrm{m}\right)$. The Streicker Bridge project has broad research and education aims, but only a part of the research relevant to the topic of this paper is presented here. More details about the other aims of the project and the monitoring system can be found in literature $[13,26]$. The aim of this section is to verify if the FE models can reproduce the results obtained by sensors that have different gauge lengths installed in real, on-site conditions. Since the deployed sensors have very low stiffness, the strain transfer from the hardened concrete to sensors is very good [10]. That is why the research focuses to analysis of dormant period of concrete, i.e. before the hydration of cement started. Once the hydration started, the 
Influence of mechanical and geometrical properties of embedded long-gauge strain sensors to the accuracy of the strain measurement

1 thermal actions create very complex strain fields that are difficult to analyze at the scale of this study.

2 Thus, this study is limited to the first 90 minutes that followed the embedding.

3 In order to assess the influence of the gauge length to the average strain measurement, three sensors: $A B_{l}$,

4

5

6

7

8

9

10

11

12

13 $A B_{2}$, and $A B_{3}$, with gauge lengths $L_{A B I}=0.3 \mathrm{~m}, L_{A B 2}=0.6 \mathrm{~m}$, and $L_{A B 3}=1.2 \mathrm{~m}$ were embedded in concrete close to each other, at the same distance from the centre of the gravity, parallel to the elastic line of the beam, in the zone with constant cross-section, as shown in Figure A.3. The view to sensors $A B_{2}$ and $A B_{3}$ before pouring of concrete is shown in Figure A.4 (the sensor $\mathrm{AB}_{1}$ is behind the two other sensors).

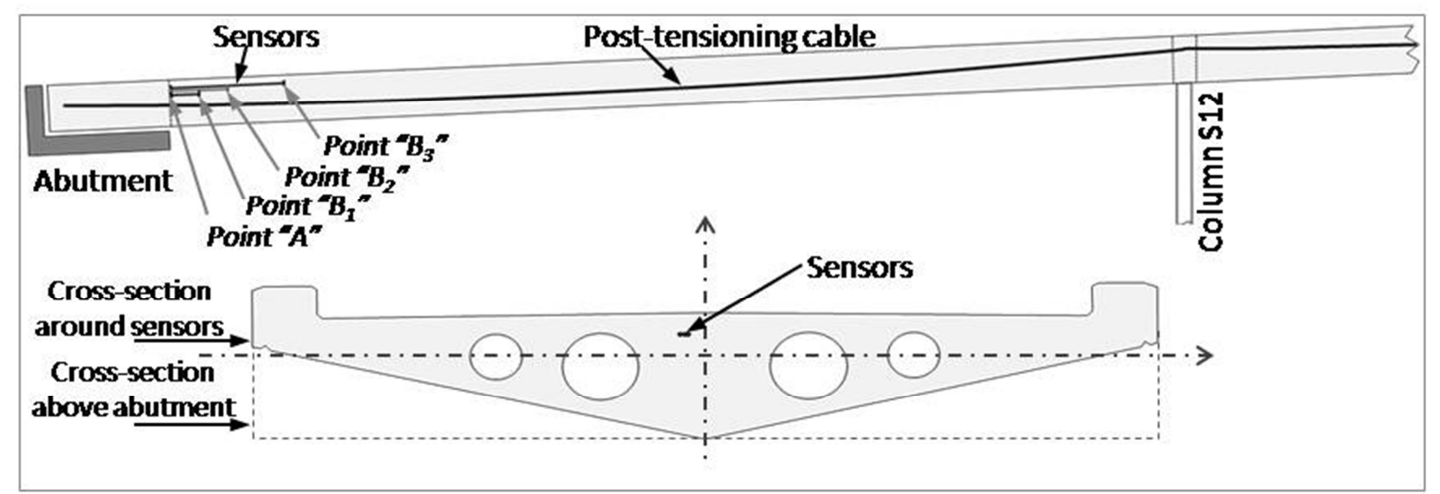

Figure A.3. Position of the sensors in the structure.

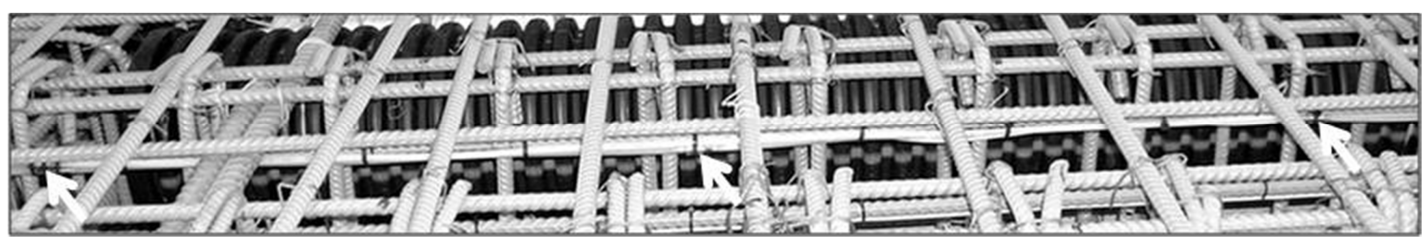

Figure A.4. Sensors $\mathrm{AB}_{2}$ and $\mathrm{AB}_{3}$ before pouring of concrete; anchoring points are indicated with arrows.

The sensors were loosely attached to rebars before the pouring to secure their position. Previous study has shown that for this type of attachment the influence of the proximity of the rebars to measurement of the sensor is negligible [10]. The geometrical and mechanical properties of the sensors are given in Tables 23. Besides the mechanical properties included in the tables, the coefficients of thermal expansion of the sensor $\left(\alpha_{t, s}\right)$ and of the concrete at very early ages $\left(\alpha_{t, h}\right)$ are also of importance. According to [10] the following coefficients can be adopted:

- Sensor (material Polyethylene):

$$
\begin{aligned}
& \alpha_{T, s}=200 \cdot 10^{-6}\left(1 /{ }^{\circ} \mathrm{C}\right) . \\
& \alpha_{T, m}=20 \cdot 10^{-6}\left(1 /{ }^{\circ} \mathrm{C}\right) .
\end{aligned}
$$

- Host material (concrete at very early age):

Pouring of the concrete was performed on October 23, 2009 at 8:47 am. The dormant period of concrete is determined to be 90 minutes based on temperature measurements, i.e., until 10:17 am. During the dormant period there were no loads applied to concrete, however the strain was generated as the 
Influence of mechanical and geometrical properties of embedded long-gauge strain sensors to the accuracy of the strain measurement

1 temperature of concrete tended to equalize with the ambient temperature, i.e. dropped for $0.75^{\circ} \mathrm{C}$. This 2 thermal strain was converted into equivalent mechanical strain for the purposes of analysis (see Equation 3 A.1). The thermal contraction of concrete at the location of sensors was expected to be uniform, and if the strain transfer from concrete to the sensors is perfect, then the three sensors would have measured similar values. However, the three sensors measured significantly different strains, which indicated that the strain transfer was not perfect, and the length of the sensors influenced the measurement.

Three FE models were created in order to reproduce the observed behavior of the sensors. Since there were no loads applied and the chemical reactions due to hydration did not induce volume changes at that stage (dormant period), it was assumed that the strain experienced by sensors is caused by temperature variation, which effect was converted in equivalent mechanical strain and introduced in the FE models by inserting thermal stresses, $\Delta \sigma_{T}$ :

$$
\Delta \sigma_{T, s}=\alpha_{T, s} \Delta T E_{s} ; \Delta \sigma_{T, m}=\alpha_{T, m} \Delta T E_{m}
$$

where $\Delta T$ is the temperature variation at very early age.

The mechanical properties of the concrete at very early age can be assumed to be similar to those of a loose-medium dense silty sand or sandy silt. It was hence assumed that they range between the limits given in Table 1. These limits fall within the typical range for loose-medium sands [27]. Both axial and planar symmetries were considered, and the FE model was set as follows:

- To reproduce the real conditions, the FE model was confined at the bottom and vertical boundaries. Vertical boundary contours have horizontal constraints whereas the bottom boundary had vertical constraints; the dimensions of the specimens have been considered large enough $(0.5 \mathrm{~m}$ wide and $2 \mathrm{~m}$ high) so that the boundaries do not influence the behavior of the sensor or the surrounding material;

- A "smooth" interface is used at the contact between the anchor piece and the concrete, while the interface between the polyethylene sensor part and the concrete was kept "non-frictional" (see Section $3)$;

- Geometrical and mechanical properties of the sensors are given in [10]; the properties of the concrete at very early ages can be assumed similar to those of a loose-medium dense silty sand or sandy silt, as presented in Tables 1-3 [27]; properties of the interface elements are given in Section 3.3.

Figure A.5 shows the mesh, geometrical properties, and interface properties used in the FE model. Comparison between the FE modeling results and the measurements is given in the same figure. The absolute difference between FE model and measurements was $5 \mu \varepsilon$ which was close to the error limit of the sensor $(4 \mu \varepsilon)$; hence, the comparison in general demonstrated that the FE model reproduced well the results obtained from on-site application, and consequently, validated the FE model. 
Influence of mechanical and geometrical properties of embedded long-gauge strain sensors to the accuracy of the strain measurement

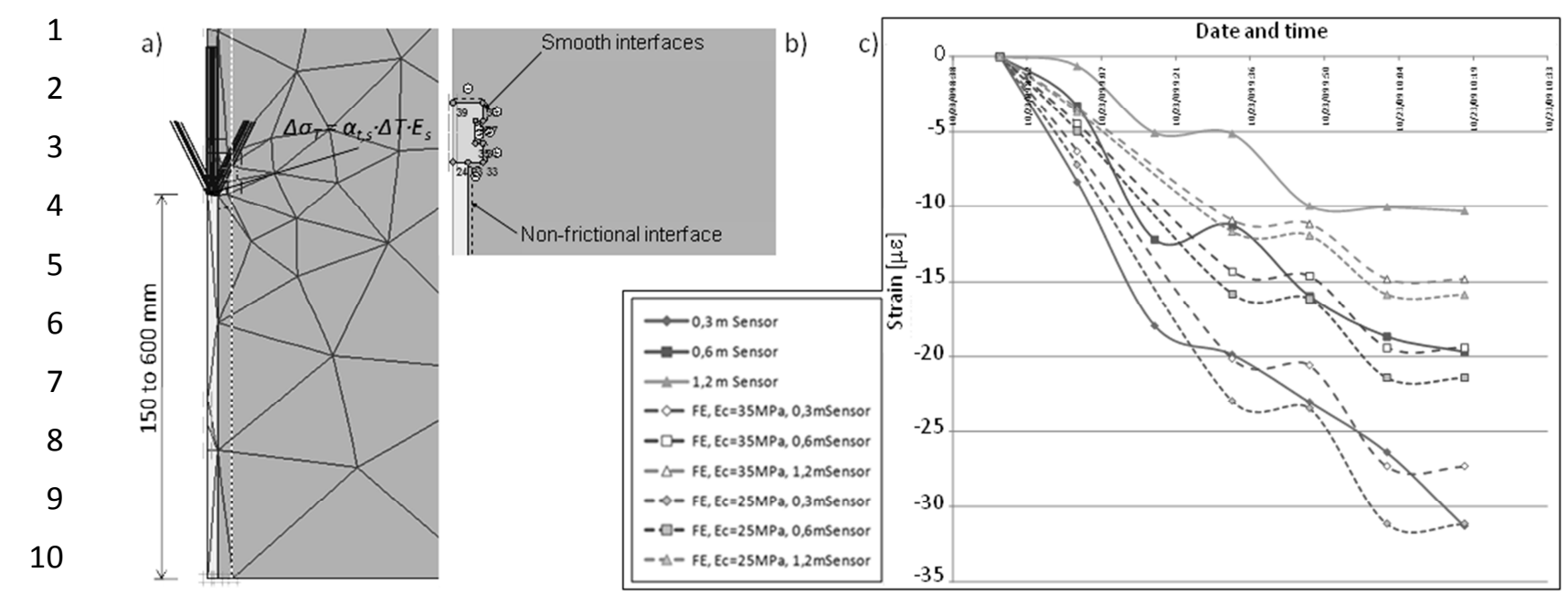

11

12

Figure A.5. a) FE model of sensors at Streicker Bridge, b) FE model - detail, c) test results vs. FE model.

13

14 